\title{
Recent advances in imaging technologies for assessment of retinal diseases
}

Taha Soomro, Neil Shah, Magdalena Niestrata-Ortiz, Timothy Yap, Eduardo M. Normando \& M. Francesca Cordeiro

To cite this article: Taha Soomro, Neil Shah, Magdalena Niestrata-Ortiz, Timothy Yap, Eduardo M. Normando \& M. Francesca Cordeiro (2020): Recent advances in imaging technologies for assessment of retinal diseases, Expert Review of Medical Devices, DOI: 10.1080/17434440.2020.1816167

To link to this article: https://doi.org/10.1080/17434440.2020.1816167

Accepted author version posted online: 04

Sep 2020.

Submit your article to this journal $₫$

Q View related articles $₫$

View Crossmark data $\asymp$ 
Publisher: Taylor \& Francis \& Informa UK Limited, trading as Taylor \& Francis Group

Journal: Expert Review of Medical Devices

DOI: $10.1080 / 17434440.2020 .1816167$

\section{Recent advances in imaging technologies for assessment of retinal diseases}

Taha Soomro ${ }^{a *}$, Neil Shah ${ }^{a}$, Magdalena Niestrata-Ortiz ${ }^{a}$, Timothy Yap ${ }^{\mathrm{a}}$, Eduardo M. Normando ${ }^{a}$, M. Francesca Cordeiro ${ }^{a}$

amperial College Ophthalmology Research Group, Western Eye Hospital, 153-173 Marylebone Road, London, NW1 5QH

*Corresponding author:

Taha Soomrot.soomro@nhs.net 


\section{Abstract}

Introduction: Retinal imaging is a key investigation in ophthalmology. New devices continue to be created to keep up with the demand for better imaging modalities in this field. This review looks to highlight current trends and the future of retinal imaging.

Areas covered: This review looks at the advances in topographical imaging, photoacoustic microscopy, optical coherence tomography and molecular imaging. There is future scoping

Expert opinion: Retinal imaging continues to develop at a rapid pace to improve diagnosis and management of patients. We will see the development of big data to gain powerful insights and new technologies such as teleophthalmology mature in the future.

Keywords: Automation, Molecular imaging, Ophthalmology, Optical coherence tomography, Retinal diseases, Retinal imaging, Teleophthalmology, Topographical imaging, Artificial intelligence

\section{Article highlights:}

- Topographical imaging such as colour fundus photography have advanced to meet the demands of diagnosis in ophthalmology, including the implementation of low-cost devices and teleophthalmology

- Optical coherence tomography (OCT) improvements have occurred in OCT angiography, swept source OCT, wide field imaging, intraoperative OCT and automation

- Molecular imaging may represent a new avenue in diagnosis of retinal pathology

- New imaging technologies will help to meet the demand to ophthalmological services worldwide 


\section{Introduction}

Ophthalmology has become one of the most imaging intensive medical specialities. This is due to an unobstructed view of the retina through the pupil, allowing imaging to be performed with relative ease, without media obstructions. Advances with retinal imaging have been coupled with advances in our understanding of retinal pathology. (1) From using basic cameras and borrowing technology used in astronomy, to developing specialised devices for the field, progress in retinal imaging has been fast paced. (2) In fact, technologies such as optical coherence tomography (OCT), which was initially created for use in ophthalmology, has been applied to other medical specialities such as dermatology and gastroenterology as well as non-medical fields such as metrology. $(3,4)$

Due to the sheer number of patients seen and the amount of retinal imaging undertaken, we have amassed amount of patient data. (5) We are seeing the use of big data and artificial intelligence in ophthalmology to aid diagnosis, management and potential treatment of retinal pathologies. $(6,7)$ Due to the ubiquity of smartphones, personalised healthcare services are also being developed for the public to use, analogous to the rise of personal banking applications. Like all other technologies, retinal imaging devices are continually being miniaturised and have the potential of being available on all our smart devices and wearables. $(8,9,10)$

The future points to remote imaging for retinal pathologies, with individual access to personalised data and greater portability of imaging technologies available.

This review looks to highlight the recent advances in imaging technologies for retinal diseases and what the future may hold.

\section{Literature review}

The initial literature review was undertaken by Dr Soomro, Dr Shah and Dr Niestrata-Ortiz. It was performed using the PubMed Central search engine using the PMC Advanced Search Builder. The topics of topographical imaging, photoacoustic microscopy, optical coherence tomography, molecular imaging and automation, reducing cost and increasing availability were reviewed. These topics were decided upon after discussion amongst the group of the most relevant areas of recent retinal imaging advances. After the initial draft, Dr Yap, Mr Normando and Professor Cordeiro contributed by adding to relevant sections and revising the manuscript.

\section{Topographical imaging}

\subsection{Colour fundus photography}

The initial impetus for retinal imaging began with the development of electronic flashes and $35 \mathrm{~mm}$ cameras, which allowed fundus photography (FP) to be performed at the beginning of the $19^{\text {th }}$ century. (2) Any retinal pathology can be initially assessed with the use of colour fundus photography. It also provides a permanent record of this finding and can be used to monitor the progress of an individual's retinal disorder. 
Limitations of FP are that it provides limited functional information about the retina, with an inability to assess the deeper layers of the retina. Optical aberrations as well as media opacities, such as cataract, can affect image quality.

FP has continued to be refined as a technology, from moving from analogue to digital which has supported the use of stereoscopic techniques (see figure 1.), as well as the ability to take images through non dilated pupils. (2)

Due to the widespread availability of smartphone technology, with small optics and related camera sensors, a lot of miniaturised devices have become available which allow users to take images of the retina and optic nerve. Initially, using simple tools such as a condensing lens, mydriatics and a smartphone camera with a co-axial light source, retinal images could be taken. (9) More sophisticated methods have now been applied. Devices such as PEEK retina vision (PEEK vision Ltd, Barbican, London) and the D-EYE digital ophthalmoscope (DEYE Srl, Padova, Italy) provide an attachment which allows a smartphone to take suitable fundal photos, through non dilated and dilated pupils. $(2,8,9)$ Now, even specific smartphones, without any attachments, can take fundus photos, due to the proximity of the rear camera with the light emitting diode $(2 \mathrm{~mm})$ allowing for an almost co-axial light source. (10)These devices have a huge potential for use in monitoring patients in the community using tele-medicine or in rural settings for ophthalmology, which PEEK vision have created infrastructure for in developing countries. (8)

\subsection{Fundus angiography}

Fundus angiography soon followed colour fundus photography in the 1960s and 1970s. (1) Novotny and Alvis were the first to produce angiograms of the chorioretinal vasculature using fluorescein (FFA). (11) Standard fundus cameras were coupled with monochromatic light filters and a contrast agent (sodium fluorescein) which was injected intravenously, allowing imaging of the retinal vasculature. This provided the ability to image different retinal pathologies such as ischaemia, where there is a lack of retinal flow, as well as vessel leakage and neovascularisation. Similar experiments were performed with indocyanine green, which is a heavier, protein bound molecule unlike sodium fluorescein, causing it to remain in the choroidal vasculature for enough time to examine the choroidal circulation. (1) This was termed IGCA.

Conventional FFA and ICGA have been essential investigations in determining the integrity of retinal and choroidal circulation respectively since they were refined for clinical use. They are important in the investigation of many common retinal pathologies such as neovascular age related macular degeneration, diabetic macular oedema, retinal vein occlusion, central serous retinopathy, as well as rare retinal dystrophies and conditions that affect other structures such as the choroid (see figure $2 \mathrm{~B}$.).

Limiting factors for these technologies are that conventional fundal angiography is an invasive procedure which can have mild (allergic reaction) to severe adverse reactions to the dye (anaphylaxis). It also provides incomplete information about the structure and function of the retina, with limited depth resolution. Similarly, to colour fundus photography, there are issues with optical aberrations and media opacities affecting the image quality of this imaging modality.

This problem has been overcome with the use of confocal scanning laser technology in current fundus photography and angiography devices such as the Heidelberg Spectralis 
(Heidelberg Engineering, Heidelberg, Germany) and Optos California systems (Optos PLC, Dunfermline, Scotland) (see figure 3.). (1)

\subsection{Confocal Scanning Laser Ophthalmoscopy}

Scanning laser ophthalmoscopy (SLO) was introduced in the 1980s. (12) SLO involves the use of point illumination with laser light, at a specific wavelength, which scans across the whole retina in a series of horizontal parallel lines. (12) This provides for an image of higher resolution and contrast, compared to traditional fundal photography, where a ring of light is used to illuminate the retina causing significant backscatter (see figure 4.). SLOs now also have a confocal aperture (cSLO) which allows light, only at a specific plane of interest, to be used to reconstruct a fundal image (13). By reconstructing fundal images across multiple planes, a three-dimensional images can be created. (12)

cSLO has allowed imaging through undilated pupils and opaque media such as cataracts using infrared light, unlike traditional fundus photography (see figure 3.A the retina is still visible through a right eye vitreous haemorrhage).

cSLO has been used in glaucoma diagnosis and monitoring for the past 15 to 20 years. Imaging and structural evaluation of the optic nerve head (rim area, rim volume, cup to disc ratio) can be performed using CSLO technology, with extrapolation of the peripapillary retinal nerve fibre layer for example with the Heidelberg Retina Tomograph (Heidelberg Engineering, Heidelberg, Germany). A recent systematic review and meta-analysis found it to have acceptable performance in diagnosing glaucomatous eyes (14). cSLO parameters have shown to correlate well with visual field defects relating to glaucoma; in particular Ahn et al. in $\mathbf{2 0 0 0}$ have highlighted cSLOs high sensitivity for detecting glaucomatous visual field defects $(89.7 \%$ in patients with a mildly impaired visual field and $100 \%$ in those with a moderately or severely impaired visual field ). (15)

Recent advances include the assessment and screening of diabetic retinopathy using CSLO based imaging. (13)

\subsection{Ultra-widefield imaging}

Conventional fundus photography, and SLO, can image 45 to 50 degrees of retina in a single frame equating to $15 \%$ of the retinal surface. (13) The shortcoming of fundus photography in capturing the peripheral retina, has been overcome with the recent advent of noncontact ultrawide field imaging (UWF), which can image $82 \%$ of the retina with a 200 -degree field of view. (16)

The most recent UWF imaging system e.g. Optos California fundus camera (Optos PLC, Dunfermline, Scotland) is a CSLO-based system which can provide ora to ora serrata imaging. It can also provide clearer images through lens opacities due to longer wavelengths of light used in image acquisition (532-nm for excitation and a 570-nm to 780-nm emission filter). (17) With an ellipsoidal mirror and CSLO, the system can take images through a nondilated pupil, in a non-contact fashion. With its increased depth of focus the system allows for simultaneous acquisition of the posterior pole and anterior retina in one picture. The system generates pseudo colour images.

UWF imaging provides panoramic images of the retina across a range of imaging modalities including pseudo colour photography (18), fluorescent angiography (FFA) (19), indocyanine 
green angiography (ICGA) (20), fundus autofluorescence (FAF) (21), optical coherence tomography (OCT) and OCT angiography (OCTA) (22).

The images can suffer from distortion in the antero-posterior (23) and horizontal axes, with stretching of the retina peripherally. The peripheral retina can also appear relatively magnified compared with the posterior pole. (18)This has been improved with new stereographic projection software algorithms (24). There can also be issues with image contrast with images taken through a miotic pupil (18) and eyelash artefacts, the latter of which can be minimised with the use of a speculum (25).

The benefit of UWF imaging over conventional colour fundus photography has been highlighted by Talks et al. in 2015, which showed that UWF imaging detected approximately $30 \%$ more neovascularisation i.e. the presence of aberrant blood vessels in the retina and optic nerve head, which tend to bleed and can become sight threatening, than standard two-field imaging when grading diabetic retinopathy (for 1562 treatment naïve eyes of patients referred from UK Diabetic Eye Screening service in England imaged with and without UWF imaging) (26).

FFA and ICGA can be performed with UWF imaging, allowing more in-depth analysis of peripheral retinal perfusion, ischaemia and neovascularisation for common disorders such as diabetic retinopathy, retinal vascular occlusions and uveitis (see figure 3B). There has also been a greater appreciation of the peripheral effects of choroidopathies such serous chorioretinopathy (CSCR), and uveitis conditions using UWF ICGCA imaging. (27) (28) (29) Fundus autofluorescence with UWF imaging can provide salient information on the peripheral changes in common conditions such as uveitis, CSCR, AMD and retinal dystrophies $(21,30,31,32,33)$.

In addition, UWF imaging can limit the need for examination under anaesthesia (EUA) for children with learning difficulties, when a good, widefield retinal image can be taken in a non-contact fashion. In children, several studies have shown the utility of UWF in a variety of disorders including childhood retinal vascular disease, retinal dystrophies, uveitis, infection, trauma, tumours, retinopathy of prematurity, and retinal detachment.

$(19,34,35,36,37,38,39)$

UWF imaging provides a reliable means of electronic documentation, with objective assessment of changes, during follow-up appointments. It can be used to improve screening and as a valuable tool for virtual clinics with telemedicine. This could potentially reduce the need for hospital appointments, as well as enable ophthalmic healthcare provision in areas with limited access to such services. $(20,40)$

\subsection{Adaptive Optics}

Traditionally higher order aberrations, and problems such as astigmatism in the optical system of the eye, have limited the transverse (lateral) resolution of fundus photography and SLO devices. This occurs due to wave front distortions created by these aberrations. $(41,42)$ This was overcome in the early 1990s when Shack Hartman wave front sensors used in astronomy, were automated and adapted for use in ophthalmology. A number of wave front sensors have since been developed to measure aberrations of the entire eye and thus allow calculation of the measured wave fronts (41). The rapid uptake of this technology in ophthalmology occurred due to its need in correcting higher order aberrations during refractive surgery. $(43,44,45)$ 
As these aberrations can be dynamic and unique to each eye, an optical element that can be reshaped into an essentially limitless number of positions is required. This is achieved using highly deformable mirrors, which can change shape by tiny electronic motor actuators on their rear surface. $(44,46)$

Applying this technology in an automated, real time fashion to existing cSLO and fundus cameras, has allowed imaging of the retina on a cellular level. (46)

In recent years, the integration of $A O$ with multiple ophthalmic imaging techniques such as optical coherence tomography (OCT) has increased both contrast and resolution with great success. Transverse resolution has improved from 10-15 $\mu \mathrm{m}$ to $\sim 2 \mu \mathrm{m}$, allowing assessment of individual retinal cell types such as retinal ganglion cells, photoreceptors and the retinal pigment epithelium (46).

AO's utility has been recognised in the early diagnosis and assessment of disease progression in inherited retinal diseases (46). In 2006, the first three dimensional observations of living human retinal cone receptors was acquired with a high-speed $A O$ spectral-domain OCT (SD-OCT) system (47). Gale et al. in 2019 (48) showed how AO automated cone measurements in subjects with retinitis pigmentosa are repeatable, as long as image quality is adequate.

AO-OCT, however, is unable to detect fluorescent signals (49). Combined with scanning laser ophthalmoscopy (SLO), AO-SLO can detect fluorescent signals, allowing retinal microvasculature and associated blood flow imaging. This has higher resolution than more conventional FFA resulting in more detailed information about the retinal capillary network and benefits from being non-invasive. Limitations at present include the small field of view obtained, meaning peripheral pathology is difficult to image (49). Newer advancements include combining AO-SLO with AO-OCT allowing high resolution and tracking capabilities (50).

\subsection{Fundus autofluorescence}

With the widespread use of fluorescein angiography, the inherent fluorescent properties of the retina without a contrast agent, when excited by light of a certain wavelength were noted and this term was coined fundus autofluorescence (FAF).

In the early 1990s Delori et al. were able to use spectrophotometry and examine excitation and emission spectra of FAF in the retina. (51) This highlighted the predominant source of fluorescence in the retina is lipofuscin, a fluorophore, which is a by-product of the visual cycle which accumulates within the retinal pigment epithelium (RPE) (52).

As several adverse effects of lipofuscin on RPE have been shown in vitro including cell membrane lysis (53), generation of free radicals (54), photo-associated complement activation (55) and photo-induced apoptosis (56), lipofuscin accumulation has been implicated in the pathogenesis of a number of retinal conditions. As a result, autofluorescence imaging is used to examine the health of the RPE and photoreceptors. $(51,57,58,59)$

SLO systems can be used for FAF with systems such as the HRA-2 blue peak autofluorescence system with an excitation wavelength of $488 \mathrm{~nm}$ (Heidelberg Engineering, Heidelberg, Germany), and Optos green autofluorescence system with an excitation wavelength of $532 \mathrm{~nm}$ (Optos PLC, Dunfermline, Scotland) (see figure 3C.). (2) Alternatively, fundus cameras, can also be used for FAF, as developed by Spaide et al. These require the addition of longer wavelength filters (excitation, $535-580 \mathrm{~nm}$ ) to overcome 
general retinal autofluorescence at all planes, particularly from macular pigment and the crystalline lens, as there are no confocal optics in the system. (60)

FAF can highlight abnormalities in autofluorescence at the macula, and in the periphery with UWF FAF, with conditions such as age-related macular degeneration, retinal dystrophies and central serous retinopathy. $(51,57,58,59)$ Areas of reduced or increased autofluorescence highlight a sick RPE, where there is an initial build-up of lipofuscin and thereafter damage to photoreceptors and loss of functional RPE.

A newer method to quantify the extent of change, or damage has been developed called quantitative fundus autofluorescence (qAF). Here FAF intensity is quantified in a sample providing an indirect measure of the extent of lipofuscin accumulation in the RPE. $(61,62)$ This is achieved by comparing the sample autofluorescence to a standardized fluorescent reference within the imaging device so the effects of variation in laser power and detector gain can be compensated for. With this FAF can then be compared over time, between eyes and between images obtained with different devices. (62) This technique enables a reliable comparison between images, including multiple examinations of the same individual at different timepoints (62), allowing assessment of disease progression and response to treatment. It has excellent repeatability and reliability (62) which was confirmed clinically by Reiter et al. in 2019 for patients with early and intermediate age related macular degeneration (AMD) (63). Apart from AMD (64), qAF has been shown to be of clinical value in retinal dystrophies such as Best's diseases (65) and Stargard's disease (66). In addition, as an indirect measure of lipofuscin density and distribution, qAF is a valuable research technique that can provide further insights into the pathophysiology of retinal conditions. (61)

\section{Photoacoustic Ophthalmoscopy}

Photoacoustic ophthalmoscopy (PAOM) works on the principle of the photoacoustic effect which was recognized by Alexander Graham Bell in the 1880s. (2) The phenomenon occurs when incident light on an object is converted from heat energy into kinetic energy, which creates a pressure wave of sound/photoacoustic wave. This has been used in photoacoustic microscopy where wideband ultrasonic waves (PA waves) are stimulated due to transient thermoelastic expansion, when a laser irradiates biological tissues (see figure 5.). The photoacoustic waves created are based on the optical absorption properties of the tissue of interest. A highspeed ultrasonic transducer can record these waves and convert this information into images. (67)

PA imaging has already been used to measure both blood oxygen saturation $\left(\mathrm{sO}_{2}\right)$ and melanin in areas of the body such as oesophagus, colon and skin, and therefore there has been investigation into its use in measuring retinal and choroidal $\mathrm{sO}_{2}$ as well as retinal pigmental epithelium (RPE) melanin. (68)

Retinal and choroidal blood $\mathrm{sO}_{2}$ and melanin (in the RPE) have high optical absorption coefficients within the visible light spectral range, so their optical absorption properties can be used to perform PAOM and measure their respective concentrations. (69) The $\mathrm{sO}_{2}$ has been shown to be abnormal in a number of ophthalmic conditions such as glaucoma (70), retinal vascular occlusion (71) and diabetic retinopathy (72). RPE melanin loss contributes to age-related macular degeneration (AMD) progression (73). 
Several animal studies have successfully used photoacoustic ophthalmoscopy (PAOM) to image the retinal and choroidal vasculature and RPE melanin $(74,75)$, showing potential uses and benefits in clinical practice. Issues with PAOM relate to the need for contact of the eye with the ultrasonic transducer, with either immersion of the eye in water or a coupling fluid, and the availability of suitable laser sources. This limit its use in the clinical setting at present.

\section{Optical coherence tomography}

\subsection{OCT}

Further advances in retinal imaging were made in 1991, by David Huang, when optical coherence tomography (OCT) was developed. His work built upon exisiting knowledge of ophthalmic interferometry. (3)

OCT can be thought of analagous to ultasonography, where instead of sound waves light is used. (3) OCT is used to create cross-sectional images of the retina (see figure 2A.). An OCT device works through an interferometer which has a reference arm with a mirror and a sample arm, which detects the light backscatter from the retina. There is a characteristic interference pattern created from light coming from the reference arm, and the tissue of interest to the sample arm which is based on the time delay between the two light waves. As one of the light waves intensity and time delay is known i.e. the reference arm, information about the light wave from the sample tissue can be extracted from the interference pattern. (3)This creates a reflectivity profile or A-scan. Several adjacent A-scans are combined to create B-scans images. (3) $(76,77)$ As light has a much shorter wavelength than sound, the resolution of OCT (less than $10 \mu \mathrm{m}$ ), is far superior to ultrasonography. The retinal images produced have been comparable to histological samples, in terms of the level of detail. (3)

Initially the technology available was time dependent and relied on the movement of the reference mirror, therefore was named time domain OCT (TD-OCT). (77) TD-OCT was limited by the speed of image acquistion and the resolution of images. There were devices such as the Stratus OCT commercially in use which were able to take around $400 \mathrm{~A}$-scans per second. Then a decade afte creating OCT, Fourier domain OCT (FD-OCT) was invented with a subset known as spectral domain OCT (SD-OCT). (77) SD-OCT works through light echo interference patterns being detected as a function of the wavelength (by Fourier transformation), by an interferometer and a stationary reference arm. There is a broadband light source and the spectral interference pattern is dispersed by a spectrometer and collected at the same time on an array detector. This technology has allowed much faster imaging as compared to TD-OCT with better resolution, with commercial devices such as the Spectralis OCT(Heidelberg Engineering, Heidelberg, Germany)and Cirrus OCT (Carl Zeiss Meditec, Jena, Germany) which take upwards of 20,000 A-scans per second. $(3,76,77)$ Optical coherence tomography (OCT) has now become an integral part of investigating patients with retinal and choroidal pathology centred around the macula, due to the limited area that can be scanned with this technology. This has been slightly improved with the advent of widefield OCT/OCTA. (22) It is used to detect evidence of fluid and structural damage, alongside the use of FFA/ICGA and colour fundus photography, in potentially sight threatening conditions such as neovascular age related macular degeneration, diabetic 
macular oedema, retinal vein occlusion, central serous retinopathy, glaucoma as well as rare retinal dystrophies and uveitis. $(78,79,80,81)$ The addition of enhanced depth imaging (EDI) counteracts the sensitivity roll-off characteristic of the technology, thereby, enabling an improved quality image acquisition of the choroid. (82)

\subsection{Swept source OCT}

Swept source OCT (SS-OCT) provides a different method for generating OCT images. A laser sweeps across a range of wavelengths. The interference pattern created from the reference and the sample arm can be recorded in almost real-time by a photodiode. (79) Therefore SSOCT technology enables the fastest scanning speed with greater than $100000 \mathrm{~A}$ scans per second. The SS-OCT uses longer wavelengths of light, with super luminescent diodes at 800$870 \mathrm{~nm}$. (79) With longer wavelengths used there is less signal strength decay (i.e. less sensitivity roll-off) versus depth which results in larger imaging ranges, allowing imaging of the vitreous, retina and choroid concurrently. So there can be enhanced imaging of the deep layers without reducing the resolution of the anterior structures, making it optimal for examination of the deeper layers of the choroid and sclera. $(79,80,83)$ It also has better penetration through media opacities. $(83,84)$ It has been shown to be superior to SD-OCT in imaging the posterior sclera (85), choroid, and important layers of the retina (inner segment (IS)/outer segment (OS) line, and external limiting membrane (ELM)) (86). This includes a better visualisation of the choroidoscleral interface, which is necessary to get a reliable measure of choroidal thickness. $(83,87,88)$

Measurements of choroidal thickness in healthy eyes, between SD-OCT with EDI and SSOCT, have been shown to have variable concordance. $(87,89,90,91)$ In patients with retinal diseases SS-OCT choroid measurements are more consistent than SD-OCT, which is thought to be due to its better ability to visualise the choroidoscleral border, which can show a greater variability in diseased eyes. $(91,92)$ Therefore SS-OCT is more useful in imaging a newly defined entity called pachychoroidopathy, i.e. choroidal thickening, which has been shown to be involved in numerous chorioretinal diseases including central serous retinopathy and polypoidal choroidal vasculopathy. (93)

\subsection{OCT Angiography}

OCT angiography (OCTA) is a new technology for imaging the vasculature of the retina and related pathology in a non-invasive manner.

OCTA uses the principles of OCT to delineate retinal blood vessels by acquiring sequential OCT B-scans in the same position to detect blood flow. (94) A decorrelation signal is created from the bulk movement of red blood cells in the retinal vasculature. (95) From this, en-face (birds eye view) images can be generated showing the different retinal and choroidal vascular plexii. The cross sectional B-scan can be correlated to the OCTA image, to find the depth and location of a vascular abnormality e.g. choroidal neovascularisation (CNV) (see figure 2C.). (94)

There are various commercial devices available which use three types of OCTA algorithms, broadly speaking, to reconstruct images of retinal vasculature: phase, intensity or both. (95) These can use spectral domain (SD-OCT) and the newer swept source OCT (SS-OCT). 
The scan dimensions can range from $3 \times 3 \mathrm{~mm}$ to $12 \times 12 \mathrm{~mm}$, with varying resolution and some newer models boast ultrawide field OCTA imaging. (95) Due to the limit of the scan dimensions this investigation is usually used to assess macular pathology.

Discerning the retinal vasculature is important for common conditions such as neovascular age related macular degeneration (nAMD) and other non nAMD related CNVs. On OCT findings these CNV usually show subretinal and/or intraretinal fluid with associated subretinal thickening, which usually corresponds to leak on traditional dye-based imaging such as FFA/ICGA. (96)

OCTA's benefits over traditional dye-based imaging is the speed of the test, repeatability to determine any changes in a vascular abnormality and that the test is non-invasive, with no associated risk of anaphylaxis. (96)

There are limitations with this technology due to motion and depth artefacts that can limit it use in diagnosis, as well its reduced ability to delineate vessels through fluid. Due to OCTA measuring the movements of red blood cells through the retinal vasculature, slow flow lesions can also be missed, such as retinal angiomatous proliferation. (97) Several studies have shown the clinical use of OCTA as compared to tradition imaging modalities such as FFA in diagnosing CNV in nAMD and other retinal pathologies like pathological myopia and central serous retinopathy (with sensitivities ranging from $50-67 \%$ and specificities from $87-91 \%)$. $(97,98,99,100,101)$ OCTA can also assess biological activity of these CNV, particularly in patients already on treatment for the condition, but is not as accurate in assessment of early disease activity. $(102,103)$ Like the traditional dye based modalities, OCTA can assess the ischaemic increase in the foveal avascular zone (FAZ) in conditions such as diabetic retinopathy, as well microvascular changes in the different vascular plexii (the superficial (SCP) and deep capillary plexus (DCP)) and the choriocapillaris around the macula relating to other retinal vascular conditions. $(96,104,105,106,107)$ Unlike traditional angiography serial imaging can be easily obtained, with the opportunity for quantitative analysis of the condition. OCTA has also been used to detect changes in uveitis conditions. It can highlight many inflammatory retinal responses such as cystoid macular oedema, optic disc oedema, vascular abnormalities in retinitis or vasculitis and inflammatory CNVs. (108)

UWF OCTA has now also become available from devices such as the Zeiss PLEX Elite 9000 (Carl Zeiss Meditec, Jena, Germany). UWF OCTA creates a 70-degree field of view, with images of a maximum size of $12 \times 12 \mathrm{~mm}$, and allows automated montaging of images to get an UWF image. (22) The benefits of this system are a better assessment of the peripheral retinal vasculature with neovascularisation or ischaemia, and the opportunity for sequential imaging for the same individual. However OCTA will still not show obvious leak and at the moment widefield SS-OCTA still requires montages to get a full peripheral view as compared to UWF-Fundus angiography (Optos PLC, Dunfermline, Scotland ). (109) UWF OCTA has shown promise in detecting common vascular abnormalities such as peripheral neovascularisation in common diseases such as proliferative diabetic retinopathy $(22,110)$, retinal vein occlusion $(111,112)$, as compared to traditional UWF FFA. $(113,114)$

\subsection{Intraoperative OCT}

Intraoperative OCT has been able to help with aspects of vitreoretinal surgery as well as for research purposes. (115) It uses the same principles of OCT technology and allows real time 
OCT peri-operatively. Standard OCTs require adequate upright patient positioning to take retinal images. In 2009, with the production of handheld OCT, the team at Duke university were able to develop intraoperative OCT. (115) From this development the new intraoperative OCT models can be handheld, fixed to the microscope or can be needle guided for retinal surgery. (116)

Handheld OCTs which can be either mounted or held directly in hand allow OCT visualisation of the retina with or without direct eye contact. These have proven particularly useful in paediatric patients, in reviewing cases such as retinopathy of prematurity, ocular albinism and retinal dystrophies. (116) The most used systems are the Bioptigen SDOIS /Envisu portable system (Bioptigen, Morrisville, NC) and the stand mounted Optovue IVue (Optovue Inc, California, USA). Handheld OCTs have issues with real time imaging in surgery, as the operating microscope cannot be used concurrently. (116)

Needle guided intraoperative OCT allows intraoperative visualisation of the retina, even through dense media, and bypasses issues with traditional OCT of tissue scatter, giving good tissue depth resolution. (117) This can allow OCT review of vitreoretinal surgery through the pars plana port approach. By integrating this system into surgical instruments, A-scans and OCT images (B-scans) can be visualised during a procedure allowing for immediate review of operative success. (116)These instruments have not been used yet clinically, as there will be the need for a helper to hold the OCT integrated instrument in the correct position whilst the surgeon operates.

The main intraoperative OCT devices that are in use during surgery are microscope integrated systems. They allows the operator to review OCTs directly whilst handling ocular tissue. These systems are either modular or fixed onto the microscope. There are three main manufacturers of these- the Zeiss RESCAN 700 (Carl Zeiss Meditec, Jena, Germany), the Haag-Streit Surgical iOCT (Haag Streit Surgical, Wedel, Germany) and the Leica Microsystems Bioptigen EnFocus (Leica Microsystems/Bioptigen, Morrisville, NC). (116) There have been two large studies- the PIONEER and follow on DISCOVER study which have looked at the feasibility of intraoperative OCT (iOCT) for retinal surgery to provide useful clinical information. The conditions that were reviewed were common vitreoretinal disorders such as macular holes, epiretinal membranes, vitreomacular traction and retinal detachments. $(118,119)$ Both studies showed specific iOCT devices to be useful in certain vitreoretinal conditions ( $60 \%$ of time valuable information was provided and $30 \%$ of the time this may have altered the surgeons decision making). $(118,119,120)$

iOCT may also prove to be beneficial in delicate sub macular surgeries such as subretinal gene therapy and subretinal protheses. (120)

There have also been recent developments in heads up display technology in vitreoretinal surgery which may look to complement intraoperative OCT in future. (120)

\section{Molecular Imaging}

An upcoming clinical technology in ophthalmology is molecular imaging. In molecular imaging, probes known as biomarkers are used to help image targets and pathways. They chemically interact with their surroundings in order to alter the image formed in the area of interest (see figure 6.). By visualising molecular processes and changes before morphological changes occur at the cellular level, disease can be identified, prevented or treated earlier (121). 
Retinal ganglion cell (RGC) dysfunction and apoptosis is observed in several ocular pathologies including glaucoma (122). A new molecular imaging technique called DARC (detection of apoptosing retinal cells) has demonstrated neuronal apoptosis in vivo for humans through the use of annexin 5 labelled with fluorescent dye DY-776 (ANX776) in a phase 1 trial with good clinical safety profile and is now being tested in a phase 2 trial (see figure 7.). $(123,124)$ Other similar techniques involve the intravitreal injection of TcapQ for in vivo detection of RGC apoptosis. However, this molecule cannot be used in humans unlike the DARC technique (125). DARC and CapQ can be used for quantitative imaging instrumentation and processing (126). By quantifying apoptotic RGCs, diagnosis and monitoring of glaucoma as well as other neurodegenerative conditions could be standardised.

Animal studies show potential to tackle the challenge of detecting subclinical retinal changes, such as the injection of $\alpha$-ICAM-1 probes to detect subtle changes in the diabetic retina pre-irreversible pathology (127). Other agents being studied in animals include HYPOX-1 and HYPOX-2 to identify hypoxic retinal tissue, often associated with diseases such as AMD and retinopathy of prematurity (128).

Nanocrystals and particles are also being developed and combined with other imaging techniques such as fluorescein angiography and OCT $(129,130)$ to target proteins such as VCAM-1 which are increased in choroidal neovascularisation.

As we move forward, toxicity studies need to be conducted to ensure a good safety profile in humans. The rapid improvement in imaging techniques, suggests ophthalmology is likely to be at the forefront of molecular techniques utilisation for clinical care (121).

\section{Automation, reducing costs and increasing availability}

The future of OCT technology and other imaging systems, for retinal imaging, will be in creating low cost devices which will allow access to these technologies in the primary care and rural settings, or allow a model of self-care. $(2,8,9,10,131)$ This will allow the further proliferation of tele-ophthalmology which has already been used with great success in the English Diabetic Eye Screening programme, when reviewing colour fundus photography, allowing the management and triage of over $83 \%$ of eligible patients with diabetes. (132) The use of artificial intelligence (AI), for grading these images, could allow even more efficient triaging of patients. Al algorithms such as IDx-DR, which have recently received FDA clearance for grading diabetic retinopathy from colour fundus images, have been shown to have a high diagnostic accuracy compared to human graders. (7) Al has also been used to automate retinal pathology detection on OCT images. The cooperation of DeepMind and Moorfields Eye Hospital has yielded an algorithm, using deep convolutional neural networks, capable of detecting retinal pathology on OCT at a comparable level to a retinal specialist (accuracy $88.4 \%-91.6 \%$ ). (6)

\section{Conclusion}

Ophthalmology as a medical field has advanced at great speed, with new imaging techniques improving our understanding and management of ocular pathology. This has occurred hand in hand with new therapeutics available in the form anti-vascular endothelial growth factor agents, as well as novel gene therapies being developed alongside stem cell 
therapies for treatment of degenerative or hereditary retinal pathologies. (2) In terms of horizon scanning, in the future we will have more accurate, multimodal imaging with better resolution of ocular structures. Quantitative, non-invasive, serial microvascular analysis, oxygenation measurement and review of real time cellular changes in patients with retinal pathologies, will be possible using the combination of technologies such as OCT angiography, photoacoustic microscopy and novel molecular imaging. Better surgical outcomes through using intraoperative OCT will be available. Using artificial intelligence, telemedicine and providing wider access to personalised smart device based imaging systems, patients will get a faster and more personalised care.

Ophthalmology currently faces a significant issue with a limited labour force and a rising burden of disease. (133)However, we can be cautiously optimistic that these advances will help us to meet this challenge and allow more patients to retain good eye health.

\section{Expert Opinion}

\subsection{Expert commentary}

The automation of retinal imaging reviews will allow a better triaging of patients to specialist services, so clinicians can deal with an increasing demand on services. This is already being implemented with the likes of FDA approved algorithms for grading diabetic retinopathy. (7) The miniaturisation of retinal imaging technologies such as OCT, ultrawide field imaging and colour fundus photography, will allow these technologies in the community and rural settings where infrastructure is lacking. $(2,8,9,10,27,131)$ Earlier diagnosis of retinal pathologies will occur, as well more timely management, with patient's being able to have imaging at a more regular frequency. Some of the new technologies will still be limited to the hospital setting but will provide new insights into eye diseases even at a pre-morbid state. Advances in colour fundus photography, OCT, OCTA, and results of analysing big data such as the UK Biobank have provided useful and sometimes surprising results. (5) The UK Biobank Eye and Vision Consortium was able to find a relationship between retinal nerve fibre layer thinning and likelihood of developing neurodegenerative conditions. (5) If we are able to detect conditions early, not only eye pathology but also other systemic conditions, then management guidelines will potentially change more towards a preventative rather than treatment strategy. For retinal pathologies this may allow more personalised and predictive treatment, rather than protocol based on a therapeutic molecule's study outcome. For example, with early pre-pathological evidence of retinal degeneration, you may monitor an individual more closely, modify their risk factors and possibly provide earlier treatment, before clinically significant retinal pathology occurs. The effectiveness of these changes in imaging technologies will be based on their accuracy and availability to use. With an increasing population of patients with eye diseases, these technologies could all allow better resource utilization and the ability to handle an increased workload. (133) These changes are already being implemented to streamline ophthalmic services, for example, with the adoption of new technologies such as OCT angiography commonplace amongst most ophthalmic centres. Newer teleophthalmology technologies, allowing remote triaging of ophthalmic conditions, such as Big Picture Medical are being trialled in centres such as Moorfields. (134)

\subsection{Key issues}


The limitations to such progress in healthcare remain in the high standards required for medical technologies to be certified to be usable in humans, the technological lag in the health sector and issues regarding privacy and confidentiality when using new cloud based digital platforms. Barriers to this technological innovation will be around costs to the healthcare sector, the ability to standardize the level of care using such systems, and healthcare as well as public adoption of using these new technologies. In the setting of the United Kingdom, there has been an injection of money into programmes that have been created to foster innovation such as the Topol fellowship together with NIHR (National Institute of Health Research) funded schemes, which have been implemented after the publication of the report on the future of healthcare in the digital age. (135) Companies from the private sector will also look to collaborate with healthcare services to provide these services. (6) National reviews from the likes of NICE UK (The National Institute for Health \& Care Excellence United Kingdom) will allow standardization of technologies. Further research being undertaken is looking at more ways to automate pathology detection in different imaging modalities, teleophthalmology services, potential automation of surgery using OCT imaging and new insights from big data. Other areas of promise will be gene therapy technologies, genomics and epidemiological studies providing novel insights into ophthalmic disease.

\subsection{Five-year review}

In 5 years' time there will be a rise in automation of retinal pathology diagnosis and a greater use of teleophthalmology services. We are already witnessing this change with the rise of virtual clinics in glaucoma and medical retina clinics. (136) Community services will have better retinal imaging modalities, and there may even be individual access to retinal imaging. There will also be more applications available to patients for regular reviews of vision assessment and therefore a more personalised care model. (8) Genetic diagnosis with rapid genome sequencing will be more readily available and will play a greater role in patient care. This will all be on the backdrop of greater pressures on healthcare services, so greater efficiency of services will be required to meet the unpresented demand. 


\section{Funding}

This paper was not funded.

\section{Declaration of Interest}

M.F. Cordeiro is a named co-inventor on granted patent EP 2231199B1 and published patent WO $2011055121 \mathrm{~A} 1$ owned by UCL and related to DARC technology. The authors have no other relevant affiliations or financial involvement with any organization or entity with a financial interest in or financial conflict with the subject matter or materials discussed in the manuscript apart from those disclosed.

\section{Reviewer Disclosures}

Peer reviewers on this manuscript have no relevant financial or other relationships to disclose. 


\section{References}

1. Keane PA, Sadda SR. Imaging chorioretinal vascular disease. Eye. 2010; 24: p. 422-427.

2. Keane PA, Sadda SR. Retinal Imaging in the Twenty-First Century State of the Art and Future Directions. Ophthalmology. 2014; 121(12): p. 2489-2500.

3. Huang D, Swanson EA, Lin CP, Schuman JS, Stinson WG, Chang W, et al. Optical coherence tomography. Science. 1991; 254(5035): p. 1178-1181.

4. Fercher AF, Drexler W, Hitzenberger CK, Lasser T. Optical coherence tomography-principles and applications. REPORTS ON PROGRESS IN PHYSICS. : p. 239-303.

5. Ko F, Muthy ZA, Gallacher J, Sudlow C, Rees G, Yang Q, et al. Association of Retinal Nerve Fiber Layer Thinning With Current and Future Cognitive Decline: A Study Using Optical Coherence Tomography. JAMA Neurology. 2018; 75(10): p. 1198-1205.

6. De Fauw J, Ledsam JR, Romera-Paredes B, al e. Clinically applicable deep learning for diagnosis and referral in retinal disease. Nature Medicine. 2018; 24: p. 1342-1350.

7. Yang WH, Zheng B, Wu MN, Zhu SJ, Fei FQ, Weng M, et al. An Evaluation System of Fundus Photograph-Based Intelligent Diagnostic Technology for Diabetic Retinopathy and Applicability for Research. Diabetes Therapy. 2019; epub ahead of print.

8. Bastawrous A, Giardini ME, Bolster NM, Peto T, Shah N, Livingstone IA, et al. Clinical Validation of a Smartphone-Based Adapter for Optic Disc Imaging in Kenya. JAMA Ophthalmology. 2016; 134(2): p. 151-158.

9. Kim Y, Chao DL. Comparison of smartphone ophthalmoscopy vs conventional direct ophthalmoscopy as a teaching tool for medical students: the COSMOS study. Clinical Ophthalmology. 2018; 13: p. 391-401.

10. Gunasekera CD, Thomas P. High-Resolution Direct Ophthalmoscopy With an Unmodified iPhone X. JAMA Ophthalmology. 2018; 29: p. 1-2.

11. Novotny HR, Alvis DR. A method of photographing fluorescence in circulating blood in the human retina. Circulation. 1961; 24: p. 82-86.

12. Pawley J. Handbook of Biological Confocal Microscopy New York, NY: Springer; 2010.

13. Goh JKH, Cheung CY, Sim SS, Tan PC, Tan GS, Wong TY. Retinal Imaging Techniques for Diabetic Retinopathy Screening. J Diabetes Sci Technol. 2016; 10(2): p. 282-294.

14. Yaghoubi M, Moradi-Lakeh M, Mokhtari-Payam M, Fakhraie G, Shokraneh F. Confocal scan laser ophthalmoscope for diagnosing glaucoma: a systematic review and meta-analysis. Asia Pac J Ophthalmol (Phila). 2015; 4(1): p. 32-9.

15. Ahn BS, Kee C. Ability of a confocal scanning laser ophthalmoscope (TopSS) to detect early glaucomatous visual field defect. Br J Ophthalmol. 2000; 84(8): p. 852-5.

16. Falavarjani KG, Wang K, Khadamy J, Sadda SR. Ultra-wide-field imaging in diabetic retinopathy; 
an overview. J Curr Ophthalmol. 2016; 28(2): p. 57-60.

17. Spaide R. Fundus autofluorescence and age-related macular degeneration. Ophthalmology. 2003; 110(2): p. 392-9.

18. Oishi A, Hidaka J, Yoshimura N. Quantification of the image obtained with a wide-field scanning ophthalmoscope. Invest Ophthalmol Vis Sci. 2014; 55(4): p. 2424-31.

19. Tsui I, Franco-Cardenas V, Hubschman J, Schwartz S. Pediatric retinal conditions imaged by ultra wide field fluorescein angiography. Ophthalmic Surg Lasers Imaging Retina. 2013; 44(1): p. 5967.

20. Nagiel A, Lalane R, Sadda S, Schwartz S. ULTRA-WIDEFIELD FUNDUS IMAGING: A Review of Clinical Applications and Future Trends. Retina. 2016. 2016; 36(4): p. 660-78.

21. Oishi M, Oishi A, Ogino K, Makiyama $Y$, Gotoh N, Kurimoto $M$, et al. Wide-field fundus autofluorescence abnormalities and visual function in patients with cone and cone-rod dystrophies. Invest Ophthalmol Vis Sci. 2014; 55(6): p. 3572-7.

22. Hirano T, KS, Toriyama Y, Nittala M, Murata T, Sadda S. Wide-field en face swept-source optical coherence tomography angiography using extended field imaging in diabetic retinopathy. $\mathrm{Br} \mathrm{J}$ Ophthalmol. 2018; 102(9): p. 1199-1203.

23. Croft D, van Hemert J, Wykoff C, Clifton D, Verhoek M, Fleming A, et al. Precise montaging and metric quantification of retinal surface area from ultra-widefield fundus photography and fluorescein angiography. Ophthalmic Surg Lasers Imaging Retina. 2014; 45(4): p. 312-7.

24. Sagong M, van Hemert J, Olmos de Koo L, Barnett C, Sadda S. Assessment of accuracy and precision of quantification of ultra-widefield images. Ophthalmology. 2015; 122(4): p. 864-6.

25. Inoue M, Yanagawa A, Yamane S, Arakawa A, Kawai Y, Kadonosono K. Wide-field fundus imaging using the Optos Optomap and a disposable eyelid speculum. JAMA Ophthalmol. 2013; 131(2): p. 226.

26. Talks SJ, Manjunath V, Steel DH, Peto T, Taylor R. New vessels detected on wide-field imaging compared to two-field and seven-field imaging: implications for diabetic retinopathy screening image analysis. Br J Ophthalmol. 2015; 99(12): p. 1606-9.

27. Friberg T, Gupta A, Yu J, Huang L, Suner I, Puliafito C, et al. Ultrawide angle fluorescein angiographic imaging: a comparison to conventional digital acquisition systems. Ophthalmic Surg Lasers Imaging. 2008; 39(4): p. 304-11.

28. Oliver S, Schwartz S. Peripheral vessel leakage (PVL): a new angiographic finding in diabetic retinopathy identified with ultra wide-field fluorescein angiography. Semin Ophthalmol. 2010; 25(1-2): p. 27-33.

29. Prasad P, Oliver S, Coffee R, Hubschman J, Schwartz S. Ultra wide-field angiographic characteristics of branch retinal and hemicentral retinal vein occlusion. Ophthalmology. 2010; 117(4): p. 780-4. 
30. Seidensticker F, Neubauer A, Wasfy T, Stumpf C, Thurau S, Kampik A, et al. Wide-field fundus autofluorescence corresponds to visual fields in chorioretinitis patients. Clin Ophthalmol. 2011; 5: p. 1667-71.

31. Pang C, Shah V, Sarraf D, Freund K. Ultra-widefield imaging with autofluorescence and indocyanine green angiography in central serous chorioretinopathy. Am J Ophthalmol. 2014; 158(2): p. 362-371.

32. Tan C, Heussen F, Sadda S. Peripheral autofluorescence and clinical findings in neovascular and non-neovascular age-related macular degeneration. Ophthalmology. 2013; 120(6): p. 1271-7.

33. Reznicek L, Seidensticker F, Stumpf C, Kampik A, Thurau S, Kernt M, et al. Systematic analysis of wide-field fundus autofluorescence (FAF) imaging in posterior uveitis. Curr Eye Res. 2014; 39(2): p. 164-71.

34. Theodoropoulou S, Ainsworth S, Blaikie A. Ultra-wide field imaging of retinopathy of prematurity (ROP) using Optomap-200TX. BMJ Case Rep. 2013.

35. Patel C, Fung T, Muqit M, Mordant D, Geh V. Non-contact ultra-widefield retinal imaging and fundus fluorescein angiography of an infant with incontinentia pigmenti without sedation in an ophthalmic office setting. J AAPOS. 2013; 17(3): p. 309-11.

36. Fung T, Muqit M, Mordant D, Smith L, Patel C. Noncontact high-resolution ultra-wide-field oral fluorescein angiography in premature infants with retinopathy of prematurity. JAMA Ophthalmol. 2014; 132(1): p. 108-10.

37. Kang K, Wessel M, Tong J, D'Amico D, Chan R. Ultra-widefield imaging for the management of pediatric retinal diseases. J Pediatr Ophthalmol Strabismus. 2013; 50(5): p. 282-8.

38. Kashani A, Learned D, Nudleman E, Drenser K, Capone A, Trese M. High prevalence of peripheral retinal vascular anomalies in family members of patients with familial exudative vitreoretinopathy. Ophthalmology. 2014; 121(1): p. 262-268.

39. Wenick A, Barañano D. Evaluation and management of pediatric rhegmatogenous retinal detachment. Saudi J Ophthalmol. 2012; 26(3): p. 255-63.

40. Afshar A, Oldenburg C, Stewart J. A Novel Hybrid Fixed and Mobile Ultra-Widefield Imaging Program for Diabetic Teleretinopathy Screening. Ophthalmol Retina. 2019.

41. Porter J. Adaptive Optics for Vision Science. En.: Wiley-Interscience; 2006. p. 63-65.

42. Hofer H, Artal P, Singer B, Arago'n JL, Williams DR. Dynamics of the eye's wave aberration. 2001; 18(3): p. 497-506.

43. Saleh M. Adaptive optics for Ophthalmology. French Journal Of Ophthalmology. 2016; 39(4).

44. Porter J. Adaptive Optics for Vision Science. En.: Wiley-Interscience; 2006. p. 33.

45. Porter J. Adaptive Optics for Vision Science. En.: Wiley-Interscience; 2006. p. 6-7.

46. Gill JS, Moosajee M, Dubis AM. Cellular imaging of inherited retinal diseases using adaptive 
optics. Eye. 2019; 33: p. 1683-1698.

47. Zhang Y, Cense B, Rha J, Jonnal RS, Gao W, Zawadzki RJ, et al. High-speed volumetric imaging of cone photoreceptors with adaptive optics spectral-domain optical coherence tomography. Opt Express. 2006; 14(10): p. 4380-94.

48. Gale MJ, Harman GA, Chen J, Pennesi ME. Repeatability of Adaptive Optics Automated Cone Measurements in Subjects With Retinitis Pigmentosa and Novel Metrics for Assessment of Image Quality. TransI Vis Sci Technol. 2019; 8(3): p. 17.

49. Zhang B, Li N, Kang J, He Y, Chen XM. Adaptive optics scanning laser ophthalmoscopy in fundus imaging, a review and update. Int J Ophthalmol. 2017; 10(11): p. 1751-1758.

50. Felberer F, Rechenmacher M, Haindl R, Baumann B, Hitzenberger CK, Pircher M. Imaging of retinal vasculature using adaptive optics SLO/OCT. Biomed Opt Express. 2015; 6(4): p. 1407-18.

51. Delori F, Staurenghi G, Arend O, Dorey C, Goger D, Weiter J. In vivo measurement of lipofuscin in Stargardt's disease--Fundus flavimaculatus. Invest Ophthalmol Vis Sci. 1995; 36(11): p. 232731.

52. Sparrow J, Gregory-Roberts E, Yamamoto K, Blonska A, Ghosh S, Ueda K, et al. The bisretinoids of retinal pigment epithelium. Prog Retin Eye Res. 2012; 31(2): p. 121-35.

53. Sparrow J, Cai B, Jang Y, Zhou J, Nakanishi K. A2E, a fluorophore of RPE lipofuscin, can destabilize membrane.. Adv Exp Med and Biol. 2006; 572: p. 63-68.

54. Boulton M, Rozanowska M, Rozanowski B. Retinal photodamage. J Photochem Photobiol B. 2001; 64: p. 144-161.

55. Zhou J, Kim S, Westlund B, Sparrow J. Complement activation by bisretinod constituents of RPE lipofuscin. Invest Ophthalmol Vis Sci. 2009; 50: p. 1392-1399.

56. Sparrow J, Nakanishi K, Parish C. The lipofuscin fluorophore A2E mediates blue light-induced damage to retinal pigmented epithelial cells. Invest Ophthalmol Vis Sci. 2000; 41(7): p. 1981-9.

57. Birnbach C, Järveläinen M, Possin D, Milam A. Histopathology and immunocytochemistry of the neurosensory retina in fundus flavimaculatus. Ophthalmology. 1994; 101(7): p. 1211-9.

58. Burke T, Duncker T, Woods R, Greenberg J, Zernant J, Tsang S, et al. Quantitative fundus autofluorescence in recessive Stargardt disease. Invest Ophthalmol Vis Sci. 2014; 55(5): p. 284152.

59. Delori F, Goger D, Dorey C. Age-related accumulation and spatial distribution of lipofuscin in RPE of normal subjects. Invest Ophthalmol Vis Sci. 2001; 42(8): p. 1855-66.

60. Schmitz-Valckenberg S, Holz FG, Bird AC, Spaide RF. Fundus autofluorescence imaging: review and perspectives. Retina. 2008; 28: p. 385-409.

61. Greenberg J, Duncker T, Woods R, Smith R, Sparrow J, Delori F. Quantitative Fundus Autofluorescence in Healthy Eyes. Invest Ophthalmol Vis Sci. 2013; 54(8): p. 5684-93. 
62. Delori F, Greenberg J, Woods R, Fischer J, Duncker T, Sparrow J, et al. Quantitative Measurements of Autofluorescence with the Scanning Laser Ophthalmoscope. Invest Ophthalmol Vis Sci. 2011; 52(13): p. 9379-90.

63. Reiter G, Told R, Baratsits M, Hecht A, Schlanitz F, Sacu S, et al. Repeatability and reliability of quantitative fundus autofluorescence imaging in patients with early and intermediate agerelated macular degeneration. Acta Ophthalmol. 2019; 97(4): p. e526-e532.

64. Orellana-Rios J, Yokoyama S, Agee J, Challa N, Freund K, Yannuzzi L, et al. Quantitative Fundus Autofluorescence in Non-Neovascular Age-Related Macular Degeneration. : p. S34-S42.

65. Sparrow J, Duncker T, Woods R, Delori F. Quantitative Fundus Autofluorescence in Best Vitelliform Macular Dystrophy: RPE Lipofuscin is not Increased in Non-Lesion Areas of Retina. Adv Exp Med Biol. 2016; 854: p. 285-90.

66. Duncker T, Tsang S, Woods R, Lee W, Zernant J, Allikmets R, et al. Quantitative Fundus Autofluorescence and Optical Coherence Tomography in PRPH2/RDS- and ABCA4-Associated Disease Exhibiting Phenotypic Overlap. Invest Ophthalmol Vis Sci. 2015; 56(5): p. 3159-70.

67. Zhang HF, Maslo K, Stoica G, Wang LV. Functional photoacoustic microscopy for high-resolution and noninvasive in vivo imaging. Nat Biotechnol. 2006; 24(7): p. 848-851.

68. Liu W, Zhang HF. Photoacoustic imaging of the eye: A mini review. Photoacoustics. 2016; 4(3): p. 112-123.

69. Jacques SL. Optical properties of biological tissues: a review. Phys Med Biol. 2013; 58(11): p. 3761.

70. Olafsdottir OB, Hardarson S H, Gottfredsdottir MS, Harris A, Stefánsson E. Retinal Oximetry in Primary Open-Angle Glaucoma. Invest Ophthalmol Vis Sci.. 2011; 52(9): p. 6409-13.

71. Eliasdottir TS, Bragason D, Hardarson SH, Kristjansdottir G, Stefánsson E. Venous oxygen saturation is reduced and variable in central retinal vein occlusion. Graefes Arch Clin Exp Ophthalmol. 2014; 253(10): p. 1409.

72. Hardarson SH, Stefánsson E. Retinal oxygen saturation is altered in diabetic retinopathy. $\mathrm{Br} \mathrm{J}$ Ophthalmol. 2011; 96(4): p. 560-563.

73. Landrum JT, Bone RA, Kilburn MD. The Macular Pigment: A Possible Role in Protection from Age-Related Macular Degeneration. Adv Pharmacol. 1996; 38: p. 537-556.

74. Liu T, H L, Song W, Jiao S, Zhang H F. Fundus Camera Guided Photoacoustic Ophthalmoscopy. Curr Eye Res. 2013; 38(12): p. 1229-1234.

75. Wei Q, Liu T, Jiao S, Zhang HF. Image chorioretinal vasculature in albino rats using photoacoustic ophthalmoscopy. J Mod Opt. 2011; 58(21): p. 1997-2001.

76. Fercher AF, Mengedoht $\mathrm{K}$, Werner $\mathrm{W}$. Eye-length measurement by interferometry with partially coherent light. Optic letters. 1988; 13(3): p. 186-188.

77. Izatt JA, Choma MA. Theory of optical coherence tomography. En Optical Coherence 
Tomography: Technology and Applications. Switzerland: Springer International Publishing; 2008. p. 47-72.

78. Ohno-Matsui K, Akiba M, Moriyama M, Ishibashi T, Tokoro T, Spaide R. Imaging retrobulbar subarachnoid space around optic nerve by swept-source optical coherence tomography in eyes with pathologic myopia. Invest Ophthalmol Vis Sci. 2011; 52: p. 9644-9650.

79. Mrejen S, Spaide R. Optical coherence tomography: imaging of the choroid and beyond. Surv Ophthalmol. 2013; 58: p. 387-429.

80. Yasuno Y, Hong Y, Makita S, Yamanari M, Akiba M, Miura M, et al. In vivo high-contrast imaging of deep posterior eye by 1-um swept source optical coherence tomography andscattering optical coherence angiography. Opt Express. 2007; 15: p. 6121-6139.

81. Ohno-Matsui K, Akiba M, Ishibashi T, Moriyama M. Observations of vascular structures within and posterior to sclera in eyes with pathologic myopia by swept-source optical coherence tomography. Invest Ophthalmol Vis Sci. 2012; 53: p. 7290-7298.

82. Spaide R, Koizumi H, Pozzoni M. Enhanced depth imaging spectral-domain optical coherence tomography. Am J Ophthalmol. 2008; 146(4): p. 496-500.

83. Waldstein S, Faatz H, Szimacsek M, Glodan A, Podkowinski D, Montuoro A, et al. Comparison of penetration depth in choroidal imaging using swept source vs spectral domain optical coherence tomography. Eye (Lond). 2015; 29(3): p. 409-15.

84. Esmaeelpour M, Povazay B, Hermann B, Hofer B, Kajic V, Kapoor K, et al. Three-dimensional 1060-nm OCT: choroidal thickness maps in normal subjects and improved posterior segment visualization in cataract patients. Invest Ophthalmol Vis Sci. 2010; 51(10): p. 5260-6.

85. Park $\mathrm{H}$, Shin $\mathrm{H}$, Park $\mathrm{C}$. Imaging the posterior segment of the eye using swept-source optical coherence tomography in myopic glaucoma eyes: comparison with enhanced-depth imaging. Am J Ophthalmol. 2014; 157(3): p. 550-7.

86. Lim L, Cheung G, Lee S. Comparison of spectral domain and swept-source optical coherence tomography in pathological myopia. Eye (Lond). 2014; 28(4): p. 488-91.

87. Adhi M, Liu J, Qavi A, Grulkowski I, Lu C, Mohler K, et al. Choroidal analysis in healthy eyes using swept-source optical coherence tomography compared to spectral domain optical coherence tomography. Am J Ophthalmol. 2014; 157(6): p. 1272-1281.

88. Copete S, Flores-Moreno I, Montero J, Duker J, Ruiz-Moreno J. Direct comparison of spectraldomain and swept-source OCT in the measurement of choroidal thickness in normal eyes. $\mathrm{Br} J$ Ophthalmol. 2014; 98(3): p. 334-8.

89. Matsuo Y, Sakamoto T, Yamashita T, Tomita M, Shirasawa M, Terasaki H. Comparisons of choroidal thickness of normal eyes obtained by two different spectral-domain OCT instruments and one swept-source OCT instrument. Invest Ophthalmol Vis Sci. 2013; 54(12): p. 7630-6.

90. Ikuno Y, Maruko I, Yasuno Y, Miura M, Sekiryu T, Nishida K, et al. Reproducibility of retinal and choroidal thickness measurements in enhanced depth imaging and high-penetration optical 
coherence tomography. Invest Ophthalmol Vis Sci. 2011; 52(8): p. 5536-40.

91. Zafar S, Siddiqui M, Shahzad R. Comparison of choroidal thickness measurements between spectral-domain OCT and swept-source OCT in normal and diseased eyes. Clin Ophthalmol. 2016; 10: p. 2271-2276.

92. Tan C, Ngo W, Cheong K. Comparison of choroidal thicknesses using swept source and spectral domain optical coherence tomography in diseased and normal eyes. Br J Ophthalmol. 2015; 99(3): p. 354-8.

93. Akkaya S. Spectrum of pachychoroid disease. Int Ophthalmol. 2018; 38(5): p. 2239-2246.

94. Cicinelli MV, Rabiolo A, Sacconi R, Carnevali A, Querques L, Bandello F, et al. Optical coherence tomography angiography in dry age-related macular degeneration. Survey of Ophthalmology. 2018; 63(2): p. 236-244.

95. Wang JC, Miller JB. For Mass Eye and Ear Special Issue: Optical Coherence Tomography Angiography: Review of Current Technical Aspects and Applications in Chorioretinal Disease. Seminars in Ophthalmology. 2019;: p. epub ahead of print.

96. Moussa M, Mahmoud L, Saad Bessa A, Lolah M, Abou Shousha M, Medhat El Hennawi H, et al. Grading of macular perfusion in retinal vein occlusion using en-face swept-source optical coherence tomography angiography: a retrospective observational case series. BMC Ophthalmology. 2019; 19(1): p. 127.

97. Coscas GJ, Lupidi M, Coscas F, Cagini C, Souied E H. OPTICAL COHERENCE TOMOGRAPHY ANGIOGRAPHY VERSUS TRADITIONAL MULTIMODAL IMAGING IN ASSESSING THE ACTIVITY OF EXUDATIVE AGE-RELATED MACULAR DEGENERATION: A New Diagnostic Challenge. Retina. 2015; 35(11): p. 2219-2228.

98. de Carlo TE, Bonini Filho MA, Chin AT, Adhi M, Ferrara D, Baumal CR, et al. Spectral-domain optical coherence tomography angiography of choroidal neovascularization. Ophthalmology. 2015; 122(6): p. 1228-1238.

99. Gong J, Yu S, Gong Y, Wang F, Sun X. The Diagnostic Accuracy of Optical Coherence Tomography Angiography for Neovascular Age-Related Macular Degeneration: A Comparison with Fundus Fluorescein Angiography. Journal of Ophthalmology. 2016;: p. epub.

100. Inoue $M$, Jung JJ, Balaratnasingam C, Dansingani KK, Dhrami-Gavazi E, Suzuki $M$, et al. A Comparison Between Optical Coherence Tomography Angiography and Fluorescein Angiography for the Imaging of Type 1 Neovascularization. Investigate ophthalmology \& vision science. 2016; 57(9): p. 314-323.

101. Querques G, Corvi F, Querques L, Souied EH. Optical Coherence Tomography Angiography of Choroidal Neovascularization Secondary to Pathological Myopia. Developments in Ophthalmology. 2016; 56: p. 101-106.

102. Faatz H, Farecki ML, Rothaus K, Gunnemann F, Gutfleisch M, Lommatzsch A, et al. Optical coherence tomography angiography of types 1 and 2 choroidal neovascularization in agerelated macular degeneration during anti-VEGF therapy: evaluation of a new quantitative 
method. Eye. 2019;: p. epub.

103. Carnevali A, Sacconi R, Querques L, Marchese A, Capuano V, Rabiolo A, et al. Natural History of Treatment-Naïve Quiescent Choroidal Neovascularization in Age-Related Macular Degeneration Using OCT Angiography. Ophthalmology Retina. 2018; 2(9): p. 922-930.

104. Choi W, Waheed N, Moult E, Adhi M, Lee B, DeCarlo T, et al. ULTRAHIGH SPEED SWEPT SOURCE OPTICAL COHERENCE TOMOGRAPHY ANGIOGRAPHY OF RETINAL AND CHORIOCAPILLARIS ALTERATIONS IN DIABETIC PATIENTS WITH AND WITHOUT RETINOPATHY. Retina. 2017; 37(1): p. 11-21.

105. Carnevali A, Sacconi R, Corbelli E, Tomasso L, Querques L, Zerbini G, et al. Optical coherence tomography angiography analysis of retinal vascular plexuses and choriocapillaris in patients with type 1 diabetes without diabetic retinopathy. Acta Diabetologica. 2017; 54(7): p. 695-702.

106. Nesper PL, Roberts PK, Onishi AK, Chai H, Liu L, Jampol LM, et al. Quantifying Microvascular Abnormalities With Increasing Severity of Diabetic Retinopathy Using Optical Coherence Tomography Angiography. Investigative Ophthalmology \& Visual Science. 2017; 58(6): p. 307315.

107. Conti FF, Qin LV, Rodrigues EB, Sharma S, Rachitskaya AV, Ehlers JP, et al. Choriocapillaris and retinal vascular plexus density of diabetic eyes using split-spectrum amplitude decorrelation spectral-domain optical coherence tomography angiography. British Journal of Ophthalmology. 2019; 103: p. 452-456.

108. Invernizzi A, Cozzi M, Staurenghi G. Optical coherence tomography and optical coherence tomography angiography in uveitis: A review. Clinical \& Experimental Ophthalmology. 2018; 47: p. 357-371.

109. Eastline M, Munk MR, Wolf S, Schaal KB, Ebneter A, Tian M, et al. Repeatability of Wide-field Optical Coherence Tomography Angiography in Normal Retina. Translational Vision Science \& Technology. 2019; 8(3): p. 6.

110. Salz D, de Carlo T, Adhi M, Moult E, Choi W, Baumal C, et al. Select Features of Diabetic Retinopathy on Swept-Source Optical Coherence Tomographic Angiography Compared With Fluorescein Angiography and Normal Eyes. JAMA Ophthalmol. 2016; 134(6): p. 644-50.

111. Kakihara S, Hirano T, lesato Y, Imai A, Toriyama Y, Murata T. Extended field imaging using swept-source optical coherence tomography angiography in retinal vein occlusion. Jpn J Ophthalmol. 2018; 62(3): p. 274-279.

112. Pellegrini M, Cozzi M, Staurenghi G, Corvi F. Comparison of wide field optical coherence tomography angiography with extended field imaging and fluorescein angiography in retinal vascular disorders. : p. epub.

113. Russell JF, Shi Y, Hinkle JW, Scott NL, Fan KC, Lyu C, et al. Longitudinal Wide Field Swept Source OCT Angiography of Neovascularization in Proliferative Diabetic Retinopathy After Panretinal Photocoagulation. Ophthalmology Retina. 2018; 3(4): p. 350-361.

114. Russell JF, Flynn Jr HW, Sridhara J, Townsend JH, Shia Y, Fana KC, et al. Distribution of Diabetic 
Neovascularization on Ultra-Widefield Fluorescein Angiography and on Simulated Widefield OCT Angiography. American Journal of Ophthalmology. 2019; 207: p. 110-120.

115. Dayani PN, Maldonado R, Farsiu S, Toth C A. Intraoperative use of handheld spectral domain optical coherence tomography imaging in macular surgery. Retina. 2009; 29(10): p. 1457-68.

116. Carrasco-Zevallos OM, Viehland C, Keller B, Draelos M, Kuo AN, Toth CA, et al. Review of intraoperative optical coherence tomography: technology and applications. Biomedical Optics Express. 2017; 8(3): p. 1607-1637.

117. Li X, Chudoba G, Ko T, Pitris C, Fujimoto JG. Imaging needle for optical coherence tomography. Opt. Lett. : p. 1520-1522.

118. Ehlers JP, Dupps WJ, Kaiser PK, Goshe J, Singh RP, Petkovsek D, et al. The Prospective Intraoperative and Perioperative Ophthalmic ImagiNg with Optical CoherEncE TomogRaphy (PIONEER) Study: 2-year results. American Journal of Ophthalmology. 2014; 158(5): p. 9991007.

119. Ehlers JP, Modi YS, Pecen PE, Goshe J, Dupps WJ, Rachitskaya A, et al. The DISCOVER Study 3Year Results: Feasibility and Usefulness of Microscope-Integrated Intraoperative OCT during Ophthalmic Surgery. Ophthalmology. 2018; 125(7): p. 1014-1027.

120. Ung C, Miller JB. Intraoperative Optical Coherence Tomography in Vitreoretinal Surgery. Seminars in Ophthalmology. 2019;: p. epub ahead of print.

121. de Carvalho JER, Verbraak FD, Aalders MC, van Noorden CJ, Schlingemann RO. Recent advances in ophthalmic molecular imaging. Surv Ophthalmol. 2014; 59(4): p. 393-413.

122. Farkas RH, Grosskreutz CL. Apoptosis, Neuroprotection, and Retinal Ganglion Cell Death: An Overview. Int Ophthalmol Clin. 2001; 41(1): p. 111-130.

123. Cordeiro MF, Normando EM, Cardoso MJ, Miodragovic S, Jeylani S, Davis BM, et al. Real-time imaging of single neuronal cell apoptosis in patients with glaucoma. Brain. 2017; 140(6): p. 1757-1767.

124. Yang E, Al-Mugheiry TS, Normando E, Cordeiro MF. Real-Time Imaging of Retinal Cell Apoptosis by Confocal Scanning Laser Ophthalmoscopy and Its Role in Glaucoma. Front. Neurol. : p. 338 epub.

125. Barnett EM, Zhang X, Maxwell D, Chang Q, Piwnica-Worms D. Single-cell imaging of retinal ganglion cell apoptosis with a cell-penetrating, activatable peptide probe in an in vivo glaucoma model. Proc Natl Acad Sci U S A. 2009; 106(23): p. 9391-9396.

126. Capozzi ME, Gordon AY, Penn JS, Jayagopal A. Molecular Imaging of Retinal Disease. J Ocul Pharmacol Ther. 2013; 29(2): p. 275-286.

127. Frimmel S, Zandi S, Sun D, Zhang Z, Schering A, Melhorn MI, et al. Molecular Imaging of Retinal Endothelial Injury in Diabetic Animals. J Ophthalmic Vis Res. 2017; 12(2): p. 175-182.

128. Evans SM, Kim K, Moore CE, Uddin MI, Capozzi ME, Craft JR, et al. Molecular Probes for Imaging 
of Hypoxia in the Retina. Bioconjug Chem. 2014; 25(11): p. 2030-7.

129. Jayagopal A, Russ PK, Haselton FR. Surface engineering of quantum dots for in vivo vascular imaging. Bioconjug Chem. 2007; 18(5): p. 1424-1433.

130. Faber DJ, de Bruin M, Aalders MCG, Verbraak FD, van Leeuwen TG. NAOMI: nanoparticle assisted optical molecular imaging. En: Nanoscale imaging, Spectroscopy, Sensing, and Actuation for Biomedical ApplicationsSan Jose: Coherence Domain Optical Methods and Optical Coherence Tomography in Biomedicine X; 2007

131. Kim S, Crose M, Eldridge WJ, Cox B, Brown WJ, Wax A. Design and implementation of a lowcost, portable OCT system. Biomedical Optics Express. 2018; 9(3): p. 1232-1243.

132. Scanlon PH. The English National Screening Programme for diabetic retinopathy 2003-2016. Acta Diabetologica. 2017; 54(6): p. 515-525.

133. Ophthalmologists TRCO. The Way Forward. London.

134. Kern C, Fu DJ, Kortuem K, Huemer J, Barker D, Davis A, et al. Implementation of a cloud-based referral platform in ophthalmology: making telemedicine services a reality in eye care. British Journal of Ophthalmology. 2019;: p. [Epub ahead of print].

135. Health Education England. The Topol Review Preparing the healthcare workforce to deliver the digital future. London.

136. Kern C, Kortuem K, Hamilton R, Fasolo S, Cai Y, Balaskas K, et al. Clinical Outcomes of a HospitalBased Teleophthalmology Service: What Happens to Patients in a Virtual Clinic? Ophthalmol Retina. 2019; 3(5): p. 422-428. 


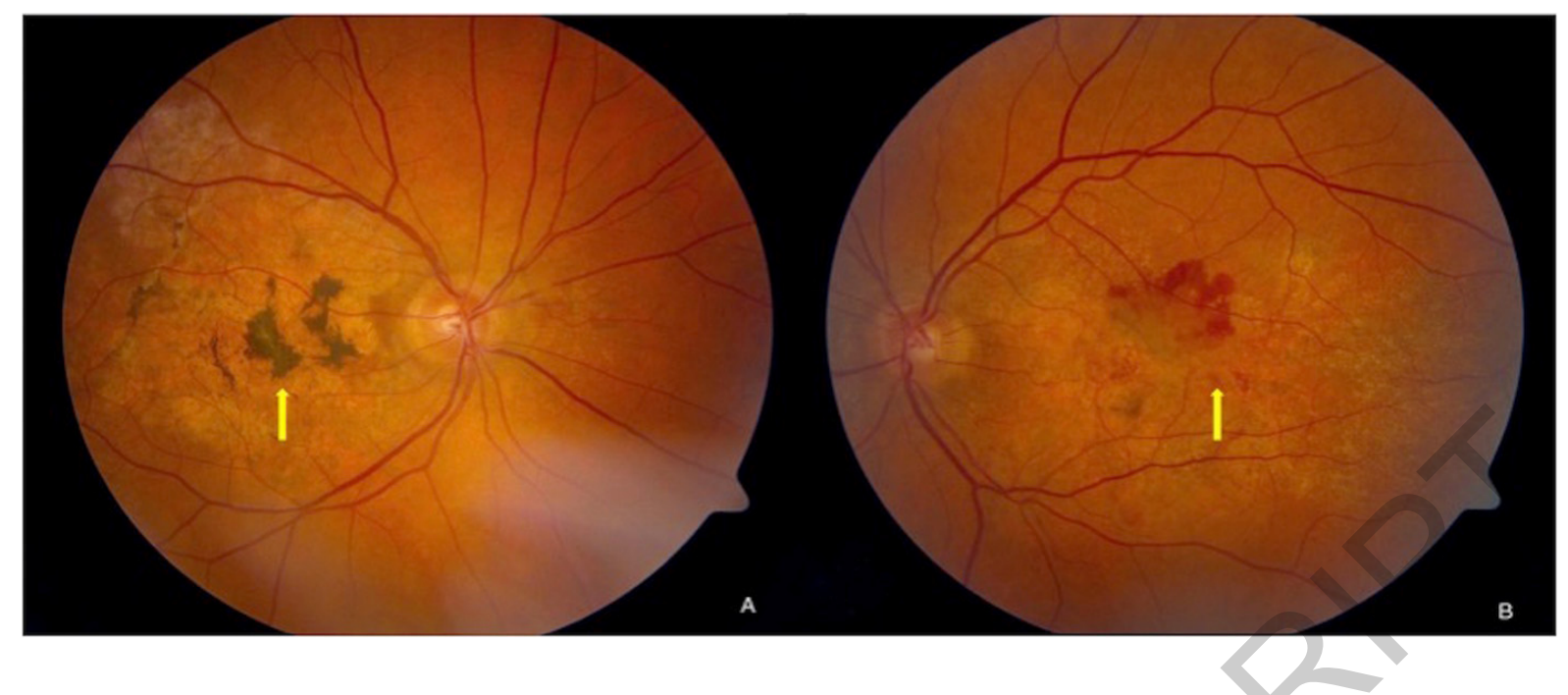

Caption: Figure 1. (A) Right eye fundal photograph witht large disciform scar over the macula (highlighted by yellow arrow) (B) Left eye fundal photograph showing numerous macular drusen and subretinal macular haemorrhage (highlighted by yellow arrow) with associated macular oedema.

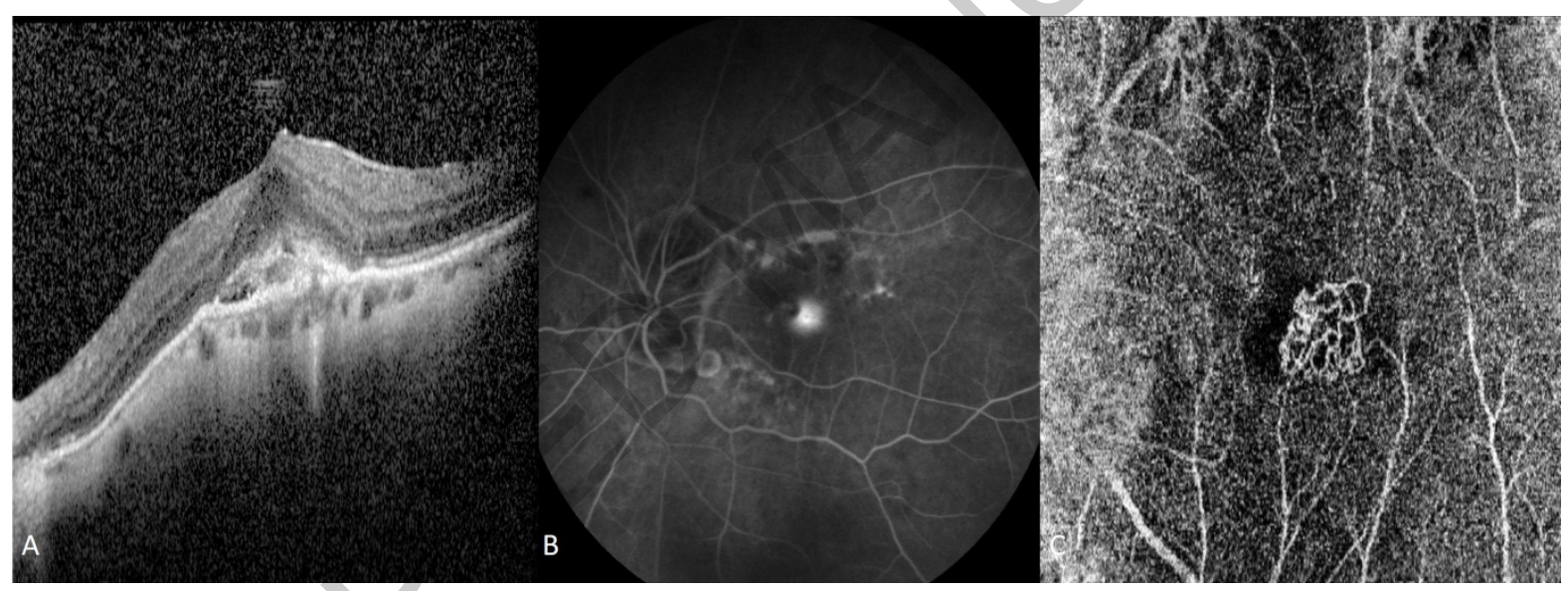

Caption: Figure 2. (A) Left eye SDOCT of patient with myopic patient with suspected myopic CNV (B) FFA showing a vague leak suggestive of myopic CNV (C) OCTA showing clear vascular network corresponding to FFA leak. 


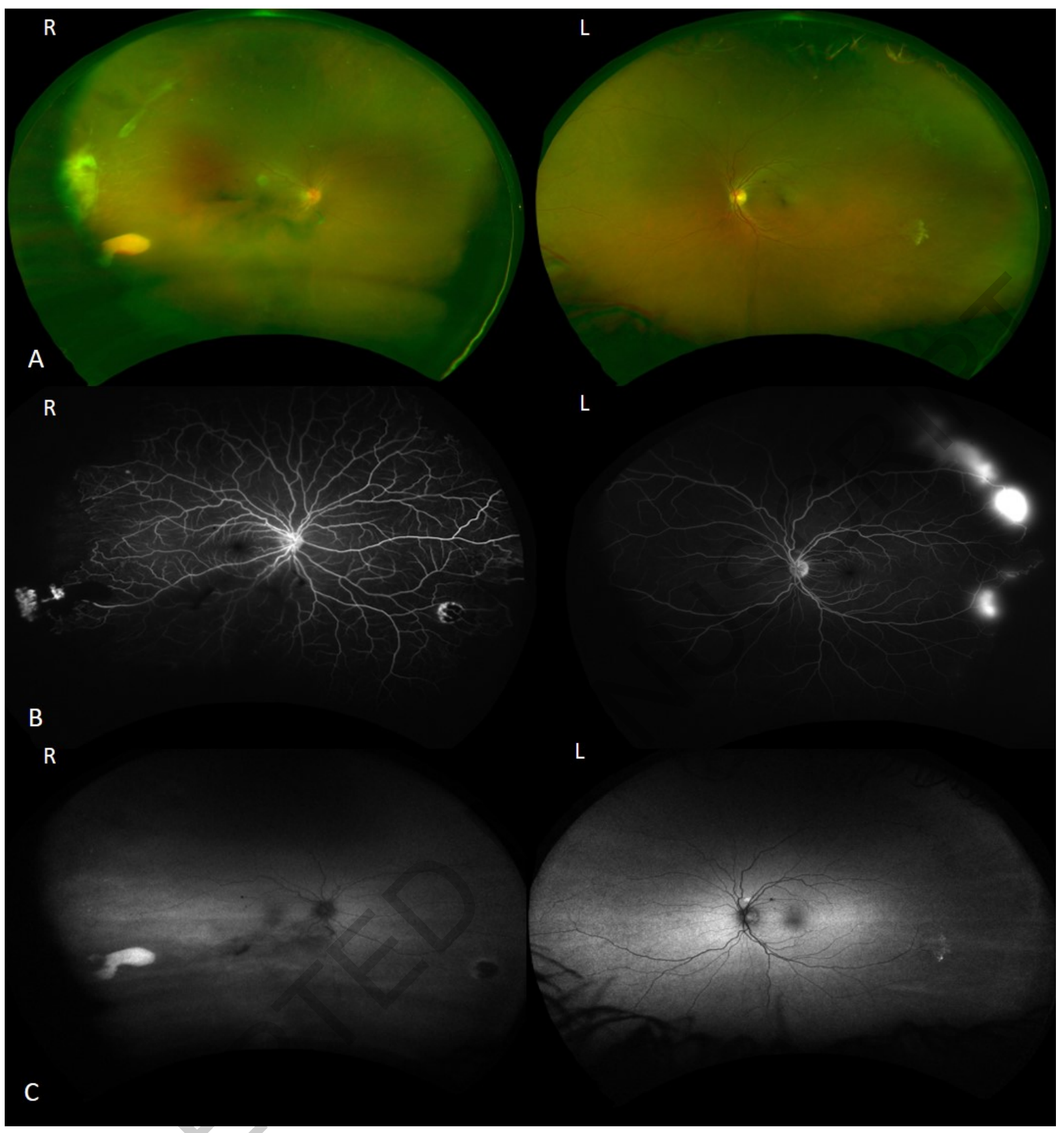

Caption: Figure 3. (A) Left and right eye pseudo-colour Optos images showing right eye vitreous haemorrhage with hazy fundal view, and left sea fan peripheral neovascularisation secondary to bilateral sickle cell retinopathy (B) Left and right fundus fluorescein angiography showing bilateral peripheral ischaemia with capillary shutdown. There is also neovascularisation in either eye, which is evidenced by areas of hyperfluorescence nasally and temporally in the right eye and temporally in the left eye (C) Fundus autofluorescence, showing masking of right eye fundus due to vitreous haemorrhage. The Optos system (Optos PLC, Dunfermline, Scotland) used has scanning laser ophthalmoscopy allowing visualisation through the right eye vitreous haemorrhage. In figure $A$ eye lashes are visible inferiorly and there is evidence of horizontal/lateral stretching of the retina with minification of the posterior pole. Optos ultrawide field imaging has multiple ultrawide field imaging modalities available (in this case pseudocolour, fluorescein angiography and fundus autofluorescence). 


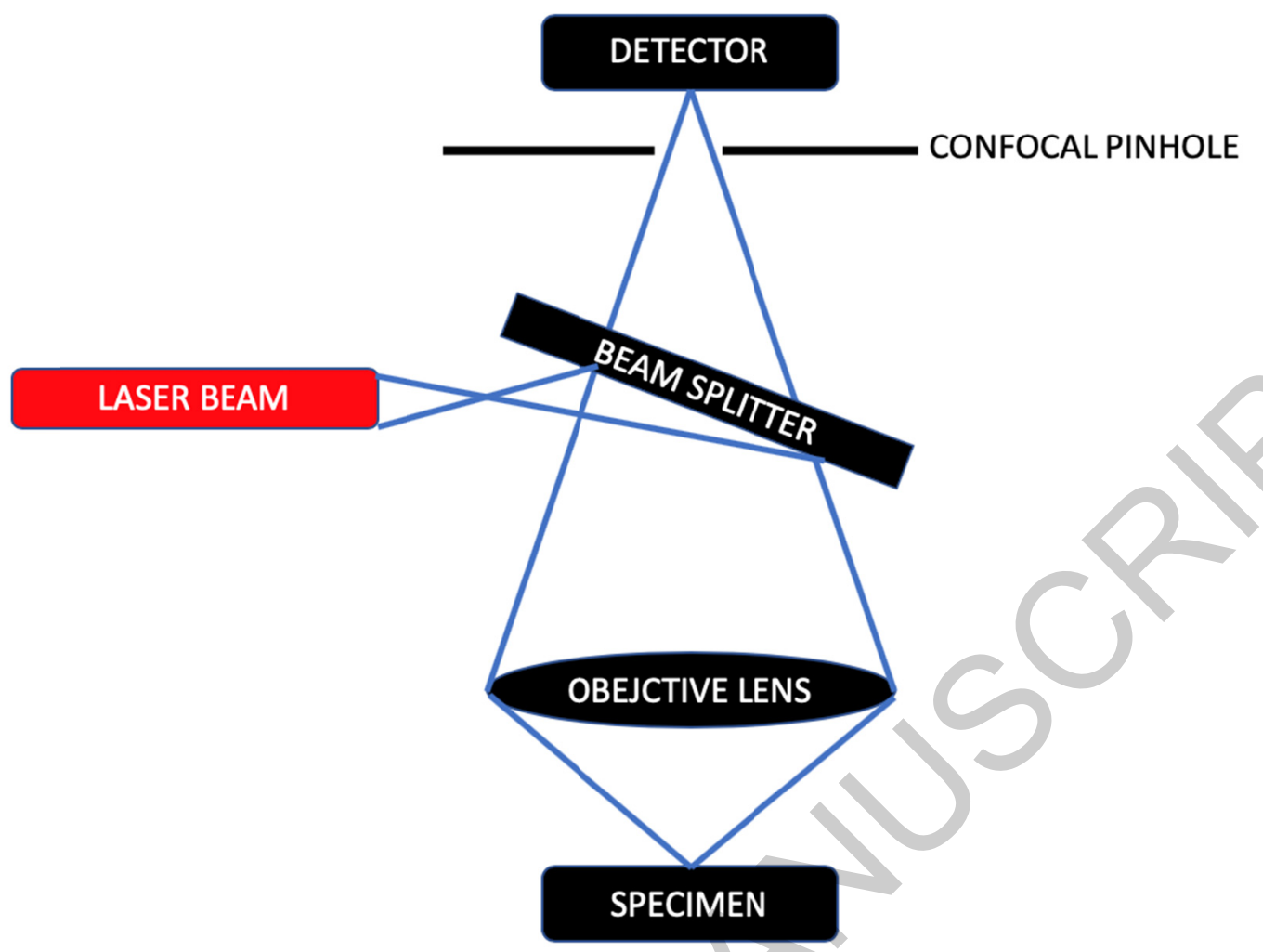

Caption: Figure 4. Schematic illustrating the principle of confocal scanning laser ophthalmoscopy (CSLO). A laser beam converges onto the focal plane within the sample, using point illumination. The light coming back from this focal plane is captured by the detector, after passing through the confocal pinhole which rejects out of focus light. 


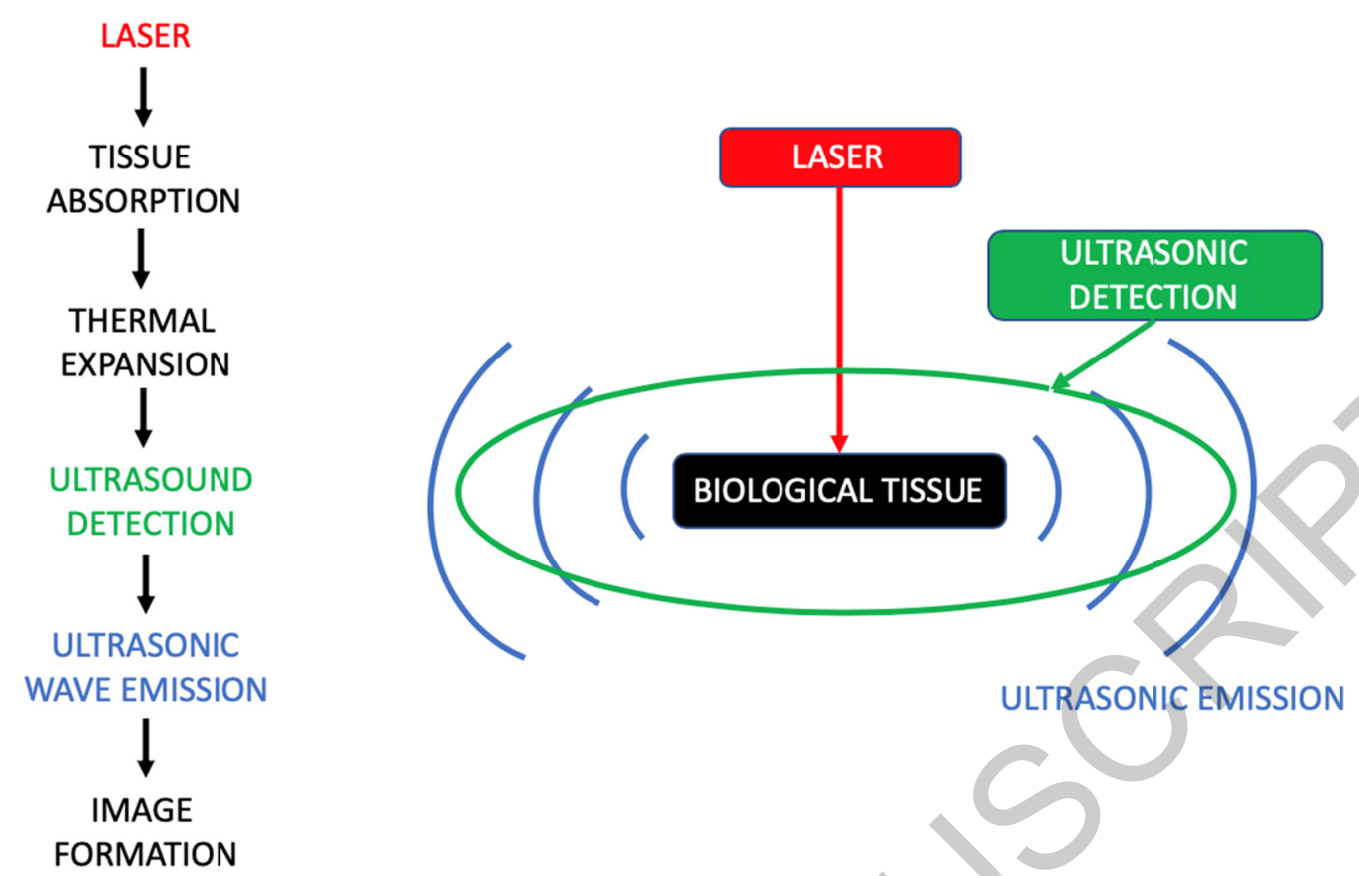

Caption: Figure 5. Schematic demonstrating photoacoustic microscopy (PA). Laser (light) absorption by biological tissue causes thermoplastic expansion resulting in ultrasonic emission (wide-band acoustic waves) that can be detected by a transducer.

\section{BIOMARKER}

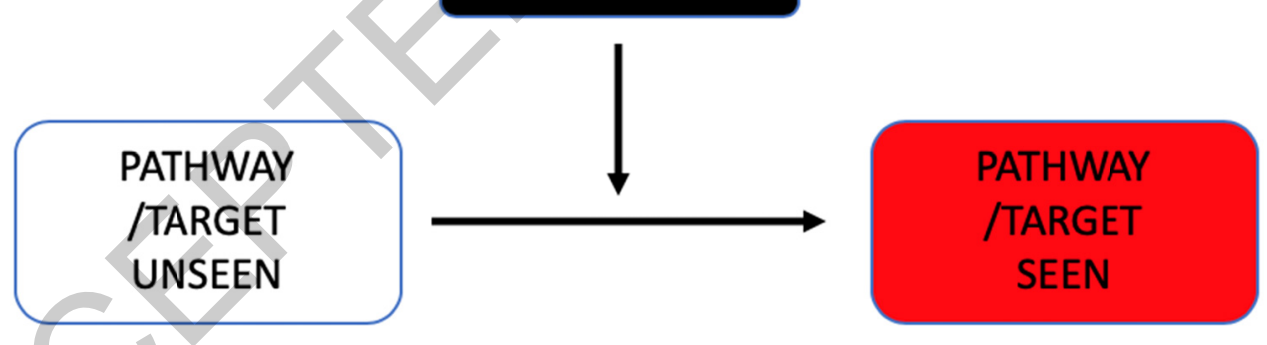

Caption: Figure 6. A simplified visual representation of the principle behind molecular imaging. Through the addition of a biomarker, pathways/targets that were previously unseen become seen. 


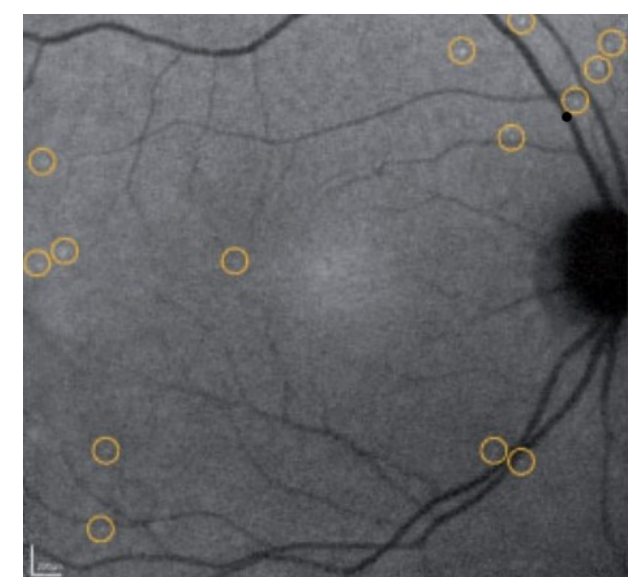

Caption: Figure 7. (With permission from Professor Francesca Cordeiro) Image of detection of apoptosing retinal ganglion cells (DARC) using fluorescently labelled annexin 5 (ANX776). The right eye posterior pole image was captured with confocal scanning laser

ophthalmoscope (HRA + OCT Spectralis, Heidelberg Engineering, Heidelberg, Germany) set to ICGA infrared fluorescence settings (diode laser $786 \mathrm{~nm}$ excitation; photodetector with $800 \mathrm{~nm}$ barrier filter). The positive spots highlight individual retinal cells with yellow rings surrounding it for demonstrative purposes. Molecular imaging tools such as DARC could have an important role to play in the future for detecting early ophthalmic disease. 